\title{
Survey on Network Mobility Support
}

\author{
Eranga Perera* \\ eranga@mobqos.ee.unsw.edu.au
}

\author{
Vijay Sivaraman ${ }^{\S}$ \\ vijay.sivaraman@csiro.au
}

\author{
Aruna Seneviratne ${ }^{\dagger}$ \\ aruna.seneviratne@nicta.com.au
}

\author{
*Department of Electrical Engineering and Telecommunications, UNSW, Sydney, Australia \\ ${ }^{\S}$ CSIRO ICT Centre, Sydney, Australia \\ ${ }^{\dagger}$ National ICT Australia (NICTA), Sydney, Australia
}

\begin{abstract}
Providing unperturbed Internet connectivity to mobile hosts has been studied in the IETF for some years now, and protocols such as Mobile IP and Mobile IPv6 have been developed. We are now witnessing the emergence of mobile networks, namely a set of hosts that move collectively as a unit, such as on ships and aircrafts. The protocols for mobility support therefore need to be extended from supporting an individual mobile device to supporting an entire mobile network. In this paper we examine the state-of-the-art in network mobility support. We first motivate the problem by considering typical network mobility scenarios and identify the characteristics that require new solutions. We then study the design requirements of the protocols that support network mobility. Thereafter, we review some of the current approaches for network mobility support, and discuss their strengths and weaknesses in addressing the design requirements. We conclude by identifying some open research issues in the realization of mobile networks.
\end{abstract}

\section{Keywords-Mobile IPv6 (MIPv6), Network Mobility (NEMO), Mobile Router (MR)}

\section{Introduction}

As wireless networking products and services proliferate, users expect to be connected to the Internet from "anywhere" at "anytime". It has moreover become the norm for a person to own more than one mobile device, say a mobile phone, a laptop and a PDA. Each of these devices could likely have multiple network interfaces that enable them to interconnect with each other as well as with other networks. These devices moving with the user together constitute a Personal Area Network or PAN, and are an example of a small scale mobile network. Access networks deployed on public transportations such as ships, trains, buses and aircrafts are examples of mobile networks at a larger scale.

The IETF has in recent years developed protocols such as Mobile IPv4 (MIP) [1] and Mobile IPv6 (MIPv6) [2] for supporting seamless connectivity to mobile hosts. These host mobility support protocols however, get triggered upon link layer handoffs which may not be seen by all nodes moving as part of a mobile network. Moreover, not all nodes in a large mobile network may be sophisticated enough to run such mobility support protocols. These deficiencies have been realized by the IETF, and a working group called NEMO (NEtwork MObility) [3] has been commissioned to extend the existing protocols or develop new ones to support network mobility in an IPv6 network.

In this paper, we survey the state-of-the-art in mechanisms for supporting network mobility. We first motivate the problem, identify its characteristics, and illustrate why existing solutions do not suffice. We next identify the solution design requirements, and then review some of the solutions proposed in the literature.

The remainder of this paper is structured as follows. Section II presents typical network mobility scenarios and identifies the most desirable architecture. Section III presents previous work in relation to the Mobile Router architecture on an IPv4 and IPv6 network mobility setting. Section IV identifies some characteristics of mobile networks and the requirements they impose on the associated design. The IETF's proposed solution is reviewed in Section V. Section VI presents some open research issues, and particular issues related to route optimization are discussed in Section VII. Section VIII lists some of the recent projects on network mobility, and Section IX concludes the paper.

\section{Mobile Network Architecture}

Consider vehicles such as ships, aircrafts and trains that contain mobile networks with a potentially large number of devices requiring global connectivity. These devices may or may not be sophisticated nodes. By employing a special entity namely a Mobile Router (MR) [4] to act as a gateway, all devices within the network can achieve global connectivity irrespective of their capabilities. NEMO Working group identifies 3 types of nodes that would be supported by a Mobile Router [5]. Local Fixed Nodes, i.e., nodes which belong to the mobile network and cannot move with respect to the Mobile Router, would typically not be able to achieve global connectivity without the support of the MR. There could also be nodes which can move with respect to the MR namely Local Mobile Nodes (home link belongs to Mobile network) and Visiting Mobile Nodes (home link does not belong to Mobile network). The mere fact that a node has MIP or MIPv6 capabilities would not guarantee session continuity for such nodes. Consider the case where the link layer handoff is via a satellite link and these nodes have no radio access capabilities to perform the necessary handoffs. In such a situation having host mobility protocols is not sufficient to maintain connectivity and would necessitate support from a MR. Also it is not efficient to expect each node to individually manage its mobility, and it would be logical to employ a MR that collectively handles the mobility of the entire mobile network. In the following, we elaborate on some of the benefits of having a network mobility architecture that relies on the MR. 


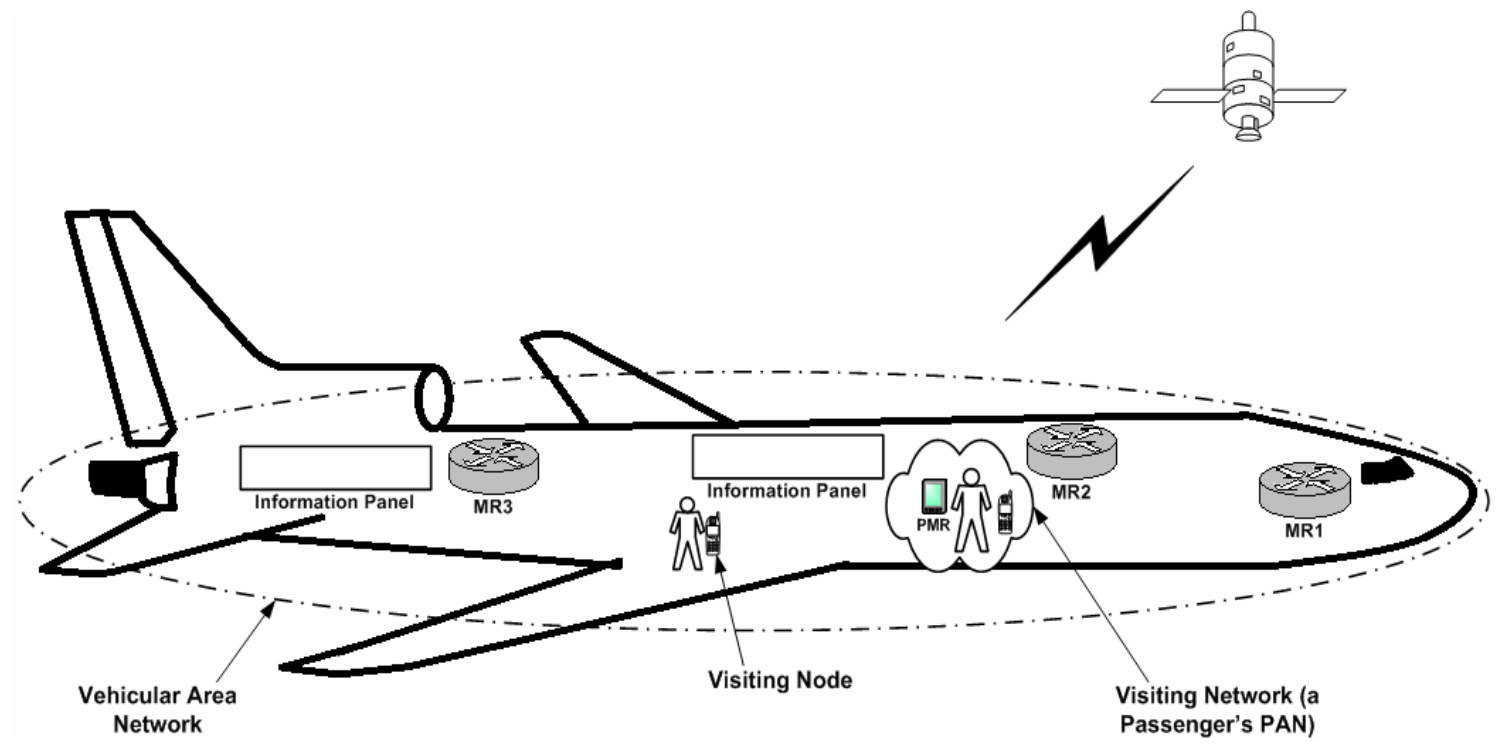

Figure 1: Mobile Network Scenario

\section{II.A. Reduced Transmission Power}

For mobile network nodes (such as on ships and aircrafts), the radio transmission distance from an onboard device to the MR is potentially much shorter than to another Access Router on the Internet. Thus by employing the MR as an Access Router, the numerous mobile network nodes need only communicate with the MR using minimal power and these nodes need not be equipped with specialized high-power communication capabilities.

\section{II.B. Reduced Hand-Offs}

Once the mobile network nodes have established a link with the MR, the link does not need to be torndown even as the mobile network moves. Since all external communication is via the MR, only the MR needs to handle link layer hand-offs. This permits unsophisticated (i.e. hand-off unaware) devices to be deployed in the mobile network, potentially yielding low-cost mobile networks.

\section{II.C. Reduced Complexity}

Once a node joins a mobile network these nodes would not have to keep changing their address since this functionality would be performed by the Mobile Router. When the mobile network changes its point of attachment to the Internet only the MR needs to auto configure a location specific address. This reduces the need for the mobile network nodes having to perform link layer handoffs as well as the need for auto configuring a new address. By having the MR perform these actions on behalf of the network nodes, the software and hardware complexity on the network nodes can be greatly reduced.

\section{II.D. Reduction in Bandwidth Consumption and Location Update Delays}

When a mobile network changes its point of attachment to the Internet, the local (fixed and mobile) nodes in the network may need to send registration messages to their Home Agents, potentially inundating the Home Agent on the home network. By having the MR send the registration updates, a single message to the Home Agent of the MR will be sufficient to register the whole network, thus offering reduction in time and bandwidth consumption. Although the Visiting Mobile Nodes would still need to send periodic registration messages to their respective Home Agents these nodes too benefit by not having to change their address whilst in the mobile network.

\section{II.E. Increased Manageability}

The MR offers an easy central point in managing the mobility features of the entire network. If protocol updates or additional features were to become necessary in the future, it is much easier to update software or policies on the MR than on each of the network nodes.

\section{II.F. Economic Incentive}

From the point of view of transportation systems, it is often commercially lucrative to provide and charge for global connectivity to passengers' mobile devices through a MR installed in the vehicle, as is being currently done by airlines.

\section{Previous Work}

Benefits of the Mobile Router architecture have been recognized as early as in the 1990s. Hager et al [6] in their paper MINT- A Mobile Internet Router describe of a router with sufficient computational power to perform all necessary communication protocol operations and enable connectivity for nodes. The MINT router provides communication software transparency and the nodes connecting to the Internet via such a router need not be modified with basic mobility support software. The use of a Mobile Router for network mobility has been specified even in the very early Request For Comments [7] on IP mobility support. In the next two subsections we present some of the research that has taken place on network mobility in an IPv4 and IPv6 setting. 


\section{III.A. MIP and Network Mobility}

Here we present a summary of how mobile networks are handled with the use of IP mobility Support Protocol (MIP). This summary follows from the IP mobility support RFC 3220 [2].

The MR would act as the foreign agent and provide a foreign agent care-of address to the mobile nodes. Packets addressed to the mobile nodes within the mobile network go through the MR's Home Agent as well as the mobile node's Home Agent. If the nodes are fixed with respect to the mobile network then MIP specifies two mechanisms that enable global connectivity for these nodes. One method is to have a permanent registration with a Home Agent to reflect the Mobile Router's home address as the fixed host's care-of address. Usually the MR's Home Agent would be used for this purpose. The second method requires the MR to advertise connectivity for the entire mobile network using normal IP routing protocols through a bidirectional tunnel to its own Home Agent.

\section{III.B. MIPv6 and Network Mobility}

Although it has been claimed that MIPv4 could support mobile networks as single mobile nodes [8], experimentations conducted in Motorola Planete Inria labs [9] have shown that this is not the case with the MIPv6 protocol. These experiments have shown that the Home Agent fails to redirect packets destined for the Local Fixed Nodes sitting behind a Mobile Router. If a packet is addressed to a Local Fixed Node the Border Router in the Home network would attempt to forward it the MR since the MR is the next hop towards the Local Fixed Node. The Home Agent acting on behalf of the MR (assuming the MR has registered its new care-of address with the HA by means of Binding Updates) would intercept the packet. Although the HA has a Binding Update for the MR it has no Binding Update for the destination address on the packet (Local Fixed Node's home address). The Home Agent being unable to handle this packet would reroute it to the Border Router. The Border Router would once again attempt to route this packet causing a repetition of the above process paving the way to a routing loop.

MIPv6 protocol's inability to handle mobile networks and the following network mobility characteristics and design requirements have paved the way to the Network Mobility Support Protocol which we present in Section V.

\section{Network Mobility Characteristics and Design Requirements}

Figure 1 depicts a typical mobile network operational scenario of a moving vehicle, for example an aircraft carrying passengers. The aircraft may be equipped with various devices, such as information panels in each cabin that provides information to the passengers, fuel sensors in the engine, and embedded sensors that gather information such as temperature, pressure and wind velocity. These devices together constitute the Vehicular Area Network (VAN). Furthermore, the passengers may carry their personal wireless devices or even an entire Personal Area Network (PAN) of devices, which enter and exit the
VAN as and when passengers embark and disembark the aircraft. A designated node in the PAN (such as the PDA denoted in Figure 1 as a PAN Mobile Router - as PMR) may act as the Mobile Router that helps connect the PAN to the VAN. The VAN is equipped with one or more Mobile Routers designated by MR1, MR2, and MR3 in Figure 1, (one MR in each cabin) that provide Internet connectivity to the nodes within the VAN. Using the above environment as an example, in the following sections we describe some of the characteristics of mobile networks and discuss how they influence the protocol design. Figure 2 illustrates an abstract view of a Vehicular Area Network.

\section{IV.A. Set of Nodes Moving as a Unit}

The defining characteristic of network mobility the notion of a set of nodes moving as a unit - is evident in the above scenario. The aircraft can be viewed as a single node changing its point of attachment to the Internet. A network mobility protocol should be able to provide global access to all the nodes within the mobile network.

\section{IV.B. Local vs. Visiting Nodes}

The mobile network in the above example includes visiting nodes, such as a passenger's wireless device, which is in a network different than its home network, as well as local nodes, such as the information panel which is within its home network. The network mobility protocol should cater to both these types of nodes.

\section{IV.C. Mobility Aware vs. Unaware Nodes}

The mobile network may contain sophisticated nodes that are "mobility aware", namely, run protocols such as MIP or MIPv6 and are able to perform link layer handoffs. However, it is quite conceivable that the mobile network may also contain nodes that are "mobility unaware", that is nodes that are not sophisticated enough to handle hand-offs. In the case of the aircraft considered above, the temperature, pressure and wind velocity information may need to be conveyed to a central database located outside the aircraft's network. Running sophisticated protocols on the low-cost sensors may be infeasible, and the network mobility support protocol should therefore be able to handle mobility on behalf of such nodes without requiring any special support from them. This goal, often termed "Network Mobility Support Transparency", is being strongly advocated by the IETF NEMO working group as very desirable when providing global connectivity to nodes within a mobile network [10].

\section{IV.D. Nested Mobility}

In the scenario considered above, the VAN of the aircraft carries within it a PAN belonging to a passenger. Where a smaller mobile network could be contained in a larger one is known as nested mobility. The network mobility protocol needs to allow for at least two levels of recursive nesting [10]. As we shall see in a subsequent section, nested networks have implications for routing and route optimization. 


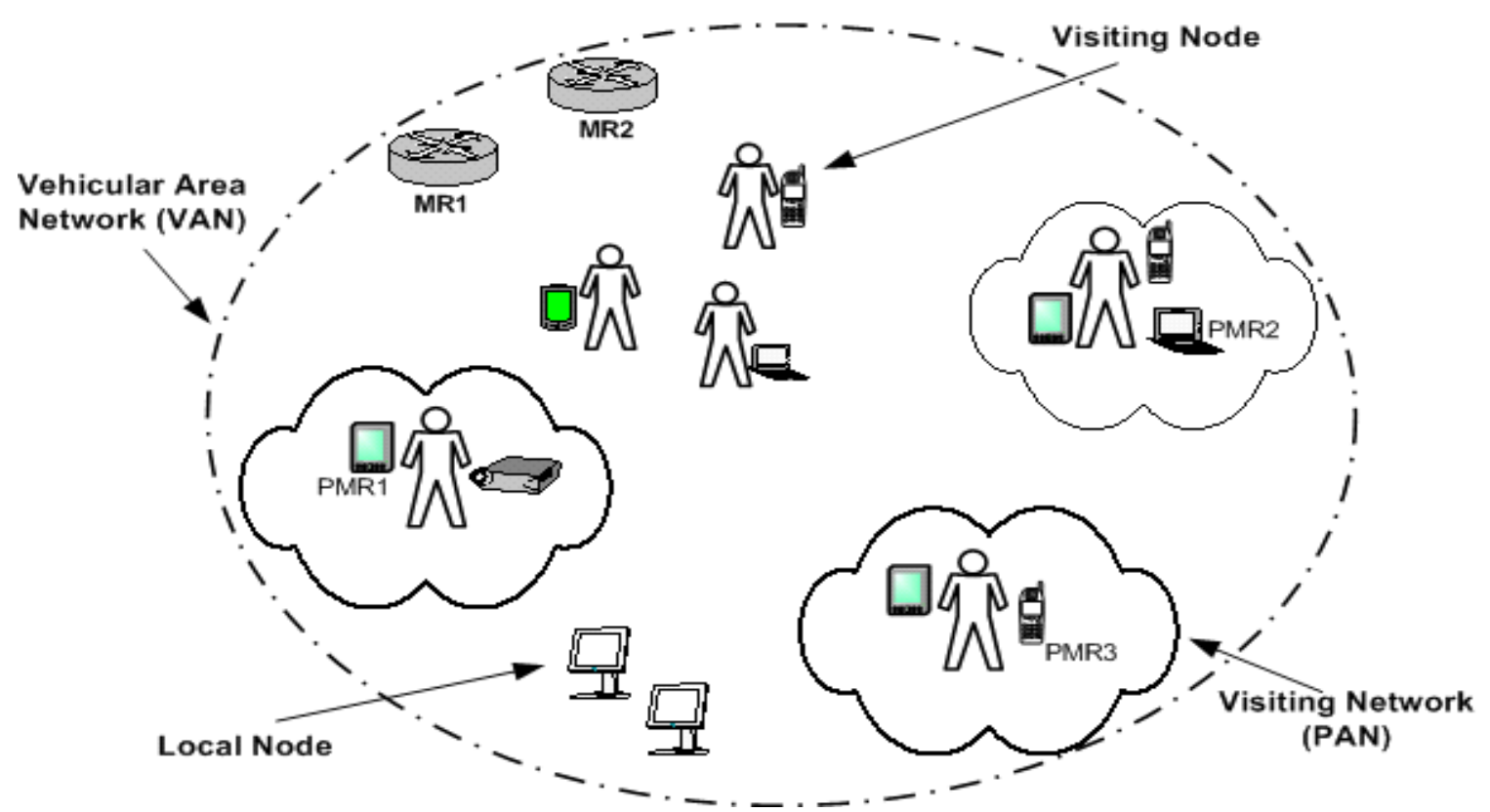

Figure 2: Abstract View of a Vehicular Area Network

\section{IV.E. Multi-Homed Mobile Networks}

The VAN in the aircraft shown above can be considered to be multi-homed if it has more than one active interface connected to the global Internet these interfaces could be through one MR or via the different MRs in the different cabins. It is desirable that the mobile network nodes be reachable even if one of the active interfaces fails. It would also be desirable to allow the mobile network nodes to attach to the active interface which suits the application it is running. For example, a passenger downloading MP3 files and listening to music may best be supported over the least congested high bandwidth link.

It is likely that VANs, such as the aircraft VAN would use different access technologies: satellites while flying and WLAN/UMTS whilst on the ground. A MR offering connectivity to the nodes sitting behind it should be able to ensure reachability to those nodes irrespective of the access technology being used.

Ernst et al [11] highlights the importance of multi homing by illustrating the benefits and goals of multi homing considering real life scenarios.

\section{IV.F. Different Sizes of Mobile Networks}

A passenger's Personal Area Network on its own with a single MR and few devices such as a PDA and a mobile phone is a very small scale network. The entire aircraft network on the other hand with a collection of subnets and a few hundred IP devices can be categorized as a large scale network. The network mobility solution should scale from small PANs to large VANs.

\section{IV.G. Disparate Handoff Rates}

Wireless cells have a limited coverage area due to limited transmission range of base stations. Mobile networks may vary significantly in the speed they move at. For example the aircraft would be stationary or taxing at very low speeds whilst on ground and when airborne would be moving at high speeds. In addition the passengers themselves may be moving within the VAN. This would result in distinct handoff frequencies and network mobility solutions have to handle this wide range of handoffs.

\section{IV.H. Mobile Devices from Different Administrative Domains}

A passenger's PAN could be from an entirely different domain from that of the mobile network. The passenger's mobile devices joining the aircraft network need to trust the MR in order to obtain Internet connectivity. This characteristic of mobile networks which requires interaction and trust among nodes from different domains has given rise to security issues unique to network mobility as opposed to host mobility in a MIPv6 network. For example Johnson et al [2] eliminated the need for a local routing proxy (Foreign Agent), which was a feature of the MIP in designing the MIPv6 protocol. This enabled the end node to handle its own routing identifier, the care-of address. In the network mobility architecture this desirable feature of MIPv6 is again compromised by having the MR as an intermediary node in the end to end communication. This requires network mobility support solutions to address specific security issues as well as comply with the standard IETF securities policies and recommendations.

\section{V.NEMO Basic Support}

The objective of the IETF NEMO working group is to develop mechanisms that provide permanent Internet connectivity to all mobile network nodes via their permanent IP addresses as well as maintain ongoing sessions as the mobile network changes its point of attachment to the Internet. Existing protocols such as MIP and MIPv6 that provide host mobility support are not sufficient due to two reasons. Firstly, not all devices in a mobile network such as the sensors on an aircraft may be sophisticated enough to run these 
complex protocols. Secondly, once a device has attached to the MR on a mobile network, it may not see any link-level handoffs even as the network moves. Thus the host mobility protocols such as MIP and MIPv6 do not get triggers indicating link-level handoffs and as a result will not initiate handover. In the network mobility architecture the MR takes care of all the nodes within the network irrespective of their capabilities.

As a first step, the IETF NEMO Working Group is developing a basic protocol [12] that ensures uninterrupted connectivity to the mobile network nodes, without considering issues such as route optimization. This protocol runs on the MR and its Home Agent (HA) and uses the same mechanisms as the host mobility protocols.

The NEMO Basic protocol requires the MR to act on behalf of the nodes within its mobile network. Firstly, the MR indicates to it's HA that it is acting as a MR as opposed to a mobile host. Secondly, the MR informs the HA of the mobile network prefixes. These prefixes are then used by the HA to intercept packets addressed to the mobile nodes and tunnel them to the MR (at its care-of address), which in turn decapsulates the packets and forwards them to the mobile nodes. Packets in the reverse direction are also tunneled via the HA in order to overcome Ingress filtering restrictions [13]. In this case the HA decapsulates the packets and forwards them to the Correspondent Nodes. This tunneling of packets is very much in the spirit of MIP and MIPv6. Note however, that NEMO differs from MIP/MIPv6 in that the MR updates the HA with the location of the entire mobile network, not just itself.

One way for the MR to update the HA is to augment the Binding Update message to include the mobile network prefix along with the MR's care-of address. This use of a Prefix Scope Binding Update (PSBU), proposed by Ernst [9], allows a single care-of address to be associated with a prefix rather than a single home address. Furthermore only a single PSBU message is required for the entire network, preserving route aggregation. Another proposal by Kniveton et al [14] uses a routing protocol between the MR and it's HA. In this case the MR need not include prefix information in the Binding Update. Petrescu et al [15] suggest the use of ICMP redirect messages to inform the HA of routes towards the MR. Alternatively, static routes could be configured to the Mobile Network via the MR's Home Address. This approach has the benefit that it does not incur additional signaling overheads. The main drawback of having static configuration is that it would prevent deployment. Another negative effect of statically configuring routes to the Mobile Network Prefixes is that these routes are present even if the related MR is unreachable. The NEMO basic support protocol adopts a flexible approach by leaving the implementers of the protocol to adopt any of the above methods when informing the HA of the routes towards the MR.

To illustrate how connectivity to mobile network nodes is achieved with the NEMO basic protocol, consider again the aircraft scenario depicted in Figure
1, with the aircraft at a foreign airport. Assume that a centralized database server needs to update the information panels in the compartments with the current times and the weather conditions of stopover cities en-route back to the home city. Assume that the Mobile Router MR2 has already registered with it's HA the VAN prefixes as well as the care-of address it obtained from the visiting network. A packet from the server sent to the home IP address of the information panel will be intercepted by it's HA. At the HA, a route lookup on the information panel's IP address will hit the subnet route for the VAN prefix, telling the HA to tunnel the packet to MR2's care-of-address. MR2 receives the packet, decapsulates it, and sends it over the air interface to the information panel. Packets in the reverse direction travel a similar route (to overcome potential Ingress filtering restrictions [13]), tunneled from MR2 via it's HA before being routed back to the Correspondent Node ${ }^{1}$.

To illustrate the communication path in the case of nested mobile networks, consider a Correspondent Node $(\mathrm{CN})$ that sends a packet to a mobile device in the passenger's PAN traveling within the aircraft's VAN. This operation of the nested mobility scenario is depicted in Figure 3. Again, it is assumed that the PAN's MR (PMR) has registered the care-of address it obtained from MR2, with it's HA (HA-PMR). Furthermore as before MR2 has registered the VAN prefixes and the care-of address with it's HA (HAMR2). A packet from a $\mathrm{CN}$ is initially addressed to the home address of the mobile device and gets routed to its home network (1). In the home network it is intercepted by the HA-PMR. A route lookup there indicates that the mobile node's prefix is at the VAN's care-of address. The HA-PMR then encapsulates and tunnels the packet to the VAN's care-of-address (2). At the VAN's home network this gets intercepted by VAN Mobile Router's Home Agent HA-MR2. Its route lookup of the VAN's care-of-address determines that the packet needs to be encapsulated and tunneled yet again to the care-of-address in the network to which the VAN is currently attached (3).

When this doubly encapsulated packet reaches MR2, which recognizes the outer care-of address as its own strips it off to reveal the care-of-address of the PMR. It sends the packet to the PMR (4), which again recognizes the (only remaining) care-of address as its own, strips it off, and sends the original packet to the mobile device in the PAN (5). Packets from the mobile device back to the $\mathrm{CN}$ traverse the same path in the reverse direction.

\section{Open Research Issues For NEMO}

The most attractive feature of the NEMO basic protocol is its simplicity, since it's a logical extension of the MIPv6 operation at the Mobile Routers and their Home Agents. The practical realization of mobile networks, nevertheless, will depend on the ability to overcome constraints posed by factors such as non optimal routing, security issues, scalability, and compatibility with the MIPv6 protocol. This section

\footnotetext{
${ }^{1}$ The operation of this scenario is not shown in Figure 3.
} 


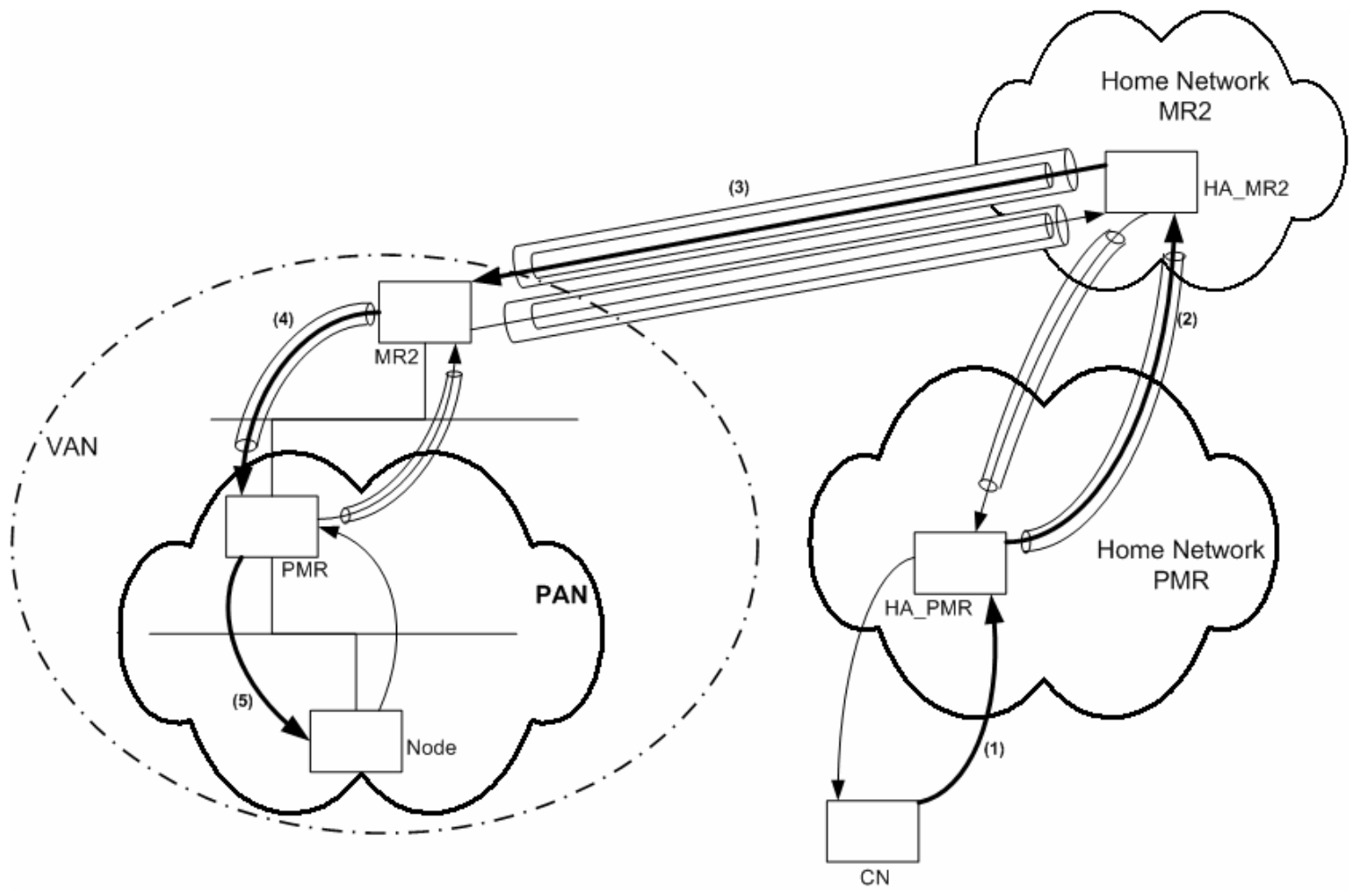

Figure 3: Nested Bi-Directional Tunneling

presents the solutions that have been proposed to tackle some of these issues and highlights areas that need further research.

\section{VI.A. Sub-Optimal Routing}

Figure 3 in Section IV illustrated how the NEMO basic protocol uses bidirectional tunneling between the MR and HA to achieve global connectivity to nodes within the mobile network. Tracing the path from a Correspondent Node to a device in the PAN, nested within the VAN illustrated how the route departs more and more from the optimal as the number of nested levels increase. This multi-angular routing issue is referred to as pinball routing [16][16]. This is highly undesirable because it would incur delays and increase the packet size at each level of nested mobility. In the next two subsections we highlight some of the side effects of sub optimal routing. The solutions that have been proposed in the literature to tackle sub optimal routing issues are discussed in section VI.

\section{VI.A.I. Increase in Packet Size}

Each added level of nested mobility requires an additional tunnel encapsulation, and these extra IPv6 headers increase the packet size and the associated overheads. Solutions that optimize the routes also reduce the levels of indirection, thereby overcoming this problem as well.

\section{VI.A.II. Performance Issues ( Delay, Packet loss)}

When a MR does a handover and changes its point of attachment it needs to activate MIPv6 and the NEMO handover procedures. Upon detection of a movement the MR obtains a care-of address from the foreign network and then indicates to its HA that it is playing the role of a MR. This handover process results in increased latency due to the multiple levels of indirection involved. The chances of packet loss are also more significant as a result of increase in latency. Research is required to adapt mechanisms such as fast handover [17] and S-MIP (Seamless MIP) [18] to support mobile networks.

\section{VI.B. Mobile Network Prefix Delegation}

One or more mobile network prefixes need to be assigned to a mobile network either statically or dynamically in order for the MR to use on the links within the mobile network. The HA assigns mobile network prefixes to the MR which are then advertised by the MR within the mobile network. Currently Mobile Network prefixes have to be assigned statically to enable network mobility. Droms [19] has proposed the reuse of the DHCPv6 prefix delegation mechanisms for a mobile network setting. Although DHCPv6 can be used there are still issues such as how to distribute keys to MRs for authentication purposes that needs to be addressed. Currently key distribution is done manually and it would be desirable to cater for MRs that would never be present at the home network in order for this manual configuration to take place.

\section{VI.C. Multi-Homing Issues}

Support for multi-homing in a network mobility environment is crucial since if a MR fails to maintain session continuity this would affect the session preservation of the entire network. Also multi-homing support would enhance the load sharing and fault tolerant capabilities of mobile networks. Policy driven decisions too can be supported by having multi-homed mobile networks. For example it would be commercially lucrative to provide Internet access to passengers according to the class of travel. 
The NEMO Basic Support protocol neither prevents nor explicitly specifies mechanisms to handle multi-homing issues. Ng et al [20][20] has analyzed the issues that arise when supporting multi-homed mobile networks by considering different configurations and scenarios. A mobile network being multi-homed due to the MR being multi-homed is one of the least complicated configurations. This case can be handled by the solutions proposed for host mobility [21] since the MR is seen as a single node in the global topology. On the other hand much research work needs to be done in order to handle multi-homed mobile networks due to multiple MRs.

The Inter Home Agents protocol (HAHA) proposed by Wakikawa et al [22] allows a Mobile Router/Mobile Node to utilize multiple Home Agents simultaneously. The adopted mechanism is to place multiple Home Agents serving the same home prefix on different links which coordinate with each other to provide Home Agent redundancy and load balancing.

In order for policy driven mechanisms supported by multi-homed mobile networks to be realized it is necessary to consider accounting issues that arise inadvertently.

Providing QOS for mobile network nodes by exploiting the redundancy provided in multi-homed mobile networks is a research area that needs further research.

\section{VI.D. Security Issues}

In NEMO the MR needs to allow subscribers from different domains to get Internet connectivity through it. In such settings where static trust relationships are lacking a variety of security threats arises. In the NEMO Basic Support protocol the use of IPsec to protect signaling messages is advocated. The protocol itself does not specify any mechanisms to handle security related issues.

Petrescu et al [23] have described the security threats related to the NEMO protocol. They have identified signaling between the MR and the HA and nested mobility configurations as two main sensitive points of the protocol. Jung et al [24] in their threat analysis draft on NEMO especially highlights issues related to IPsec and other tunneling mechanisms between the MR and the HA.

Security mechanisms for network mobility are at a preliminary stage and much work needs to be done in order for mobile networks to be deployed in a secure setting.

\section{VI.E. AAA Issues (Authentication, Authorization and Accounting Issues)}

Providing Internet access to commuters of public transportation systems would necessitate much consideration for Authentication, Authorization and Accounting issues. Access control is vital in such wireless public access networks in order for any NEMO solution to be viable.

Currently within the IETF MIPv6 Working Group there is much interest shown in adapting IPv6 AAA mechanisms for host mobility. Mechanisms to adapt the designated AAA protocol Diameter, for a MIPv6 network is currently being studied [25]. But network mobility introduces new AAA issues due to mobile network nodes relying on a previously unknown entity to take care of their mobility management tasks. Recognizing such issues Barz et al [26] have outlined a network access control model for vehicular mobile networks. Since the introduction of network access control via AAA entities causes handover delays, Barz et al [26] advocate on distributing these entities to minimize such delays.

Billing mechanisms for commuters of public transportation systems for Internet services is a very important issue that needs to be tackled from the business point of view.

\section{Route Optimization For NEMO}

The effects of pinball routing and IP header overheads could hinder the deployment of mobile networks since non optimal routing has a negative impact not only on the mobile network but on the Internet as a whole.

Providing optimal routing in a network mobility setting is not an easy task. This is due to the introduction of an intermediary node in the communication between a node inside a mobile network and a Correspondent Node. This has raised an issue in using MIPv6 route optimization mechanisms for network mobility, namely that the nodes within the mobile network are unable to perform the MIPv6 Return Routability test (RR) [2], which is needed to verify to the Correspondent Nodes that the home address and the care-of address are collocated. This is not possible since the nodes within the network do not have their own care-of addresses. If the MR performs the MIPv6 route optimization procedure on behalf of the nodes sitting behind it, then this would require extensions to the MIPv6 operation of Correspondent Nodes and severely impact the scalability of MRs. Scalability is affected because the MR is required to keep account of Correspondent Nodes and send Binding Updates to them on behalf of the nodes within the network. In a typical mobility scenario such as a public transportation system the number of Correspondent Nodes communicating with mobile network nodes could reach up to several hundreds.

Recognizing the impracticability of requiring the Mobile Router to send Binding Updates to Correspondent Nodes several route optimization solutions have been proposed. We present them in the following sections.

\section{VII.A. Route optimization without the participation of the nodes within the network}

In large Mobile networks, requiring the MR to send each Correspondent Node an individual Binding Update causes a Binding Update implosion. In order to overcome this scalability issue Ernst et al [27] have proposed a method in which the MR sends a Prefix Scope Binding Update to a multicast address to which Correspondent Nodes would have subscribed. A mobile network needs to have a multicast address which it registers with the Domain Name Server (DNS). The MR sends a periodic Binding Update 
containing the mobile network prefix and the MR's care-of address to this multicast address. Correspondent Nodes can join the multicast group using IPv6 multicast mechanisms. Although this solution is beneficial for large mobile networks with many Local Fixed Nodes it requires major changes to MIPv6 and also to the already widely deployed DNS system.

Optimized Route Cache Management protocol (ORC) [28] relies on scattering a route of a mobile network to portions of the Internet by means of Binding Routes (an association between the mobile network prefix and the care-of address) and ORC routers. Some Interior Gateway Protocol (IGP) Routers named as ORC routers are used in order to maintain a Binding Route (BR) to the mobile network persistently. Whenever the MR moves ORC routers receives a BR notification which will be cached in their routing tables. The ORC routers will advertise a proxy route to the mobile network by using IGP protocols and will capture packets destined to the mobile network. The packets will be forwarded to the care-of address on the BR, thus avoiding routing via the MR's home network. Since it is not possible to make every router on the Internet an ORC router it has been suggested that these routers be deployed in networks where there are Correspondent Nodes for the mobile network. This scheme would only provide optimal routing if ORC routers are available on the Correspondent Nodes networks.

\section{VII.B. Route optimization with the participation of the nodes within the network}

The solutions [28], [29], [30], [31], [32], [33] propose route optimization solutions with the participation of nodes within the network. The generic idea of these schemes requires the MR when in a foreign network to obtain a care-of prefix (rather than just a single address), and re advertise this location dependent prefix into the mobile network. The mobile network nodes can then auto configure a location specific careof address, which when communicated back to the correspondent nodes leads to optimal routing, as per MIPv6.

The solution proposed by Jeong et al [29] incorporates a method in order to route optimize as well as a method to allow mobile nodes to optimize their DNS name resolutions. This solution is only aimed at mobile nodes within the network.

Ohinishi et al [32] has proposed a route optimization method based on HMIPv6 [34] technology and it is evident in this scheme added functionality is required at the correspondent nodes and also from the nodes within the network.

Perera et al [33] have proposed an optimal routing architecture for network mobility named as OptiNets which caters for any type of node present within the mobile network. The main objective of the OptiNets architecture is to enable optimal routing for mobile nodes by exploiting the desirable features of the MIPv6 and the NEMO Basic Support protocol. By introducing a lightweight protocol between the Mobile Routers and their Home Agents benefits of aggregated routing is preserved as per NEMO protocol. This protocol named as Distributed Home Agent Protocol (DHAP) authorizes the Mobile Router to act as the Home Agent for the Local Mobile Nodes. This solution does not require any added functionality to MIPv6 Correspondent Nodes nor to any node within the mobile network when enabling optimal routing for all nodes in the mobile network.

\section{VII.C. Proposals specifically addressing the nested mobility issue}

Thubert et al [35] have proposed the use of a new routing header called the Reverse Routing Header $(\mathrm{RRH})$ in order to build a nested mobile network which avoids the nested tunnels overhead. The Reverse Routing Header which is similar to the MIP Loose Source Routing, records the route out of the nested mobile network. This can be converted to a routing header for packets destined to the mobile network. In order to further illustrate the RRH solution, consider the nested mobile network bidirectional tunneling depicted in Figure 3. The packets originating from a node in the PAN has to go through two home agents before reaching the Correspondent Node leading to very inefficient pinball routing. If a $\mathrm{RRH}$ is used the PMR would in addition to tunneling the packet to its Home Agent add a routing header with a predetermined number of slots. In the first slot the PMR puts its home address. This packet then has the PMR's care-of address as source address and PMR's Home Agent (HA_PMR) as the destination address. The next router (in this case last router) on the path which is MR2 notices that the packet already contains a RRH, so it overwrites the source address of the packet with its own address (MR2 care-of address) and puts the PMR's care-of address in the second slot. The outer packet now has MR2_COA as source address and HA_PMR as destination address. When the packet reaches HA_PMR, this Home Agent uses the information on the RRH in entering a binding update for the PMR's home address. Now when a packet arrives for PMR to its home network HA_PMR can trivially construct a routing header with MR2_COA and PMR_COA. This allows bypassing the home agent of MR 2 . It is evident even if there are $\mathrm{n}$ levels of nested mobility the packet would go through only a single Home Agent, bypassing (n-1) Home Agents.

$\mathrm{Ng}$ et al [36] have proposed a solution which overcomes the need to modify the IP headers in transit. Their mechanism relies on the ability of the sender to inform the recipient the global address of the Access Router to which it is attached. From this information the recipient can construct the chain of Access Routers the sender is attached to. $\mathrm{Na}$ et al [37] claim that the former solution is open to security vulnerabilities and the latter is complicated and attempt to extend the RRH solution with security in mind.

\section{NEMO Projects}

Along with the IETF NEMO working group network mobility research projects influences the evolvement of network mobility protocols. We 
consider some of these projects in the following subsections.

\section{VIII.A. eMotion (Network in Motion) child project of OCEAN (On-board Communication, Entertainment And iNformation)}

OCEAN [38] which is a University of New South Wales and Australian Research Council sponsored project with collaborators being National ICT Australia (NICTA) [39] and Boeing Airline Company was founded in 2003. This project recognizes the need for extension of Internet services for public transportation systems and encompasses two research areas namely networking and data management. The child project eMotion [40] handles networking issues pertaining to providing global Internet access to passengers via Mobile Routers and wide area wireless access systems.

\section{VIII.B. OverDRiVE (Over Dynamic multi-Radio Networks in Vehicular Environments)}

OverDRiVE [41] is an Information Society Technologies (IST) project and is ongoing work of the DRiVE [42] project. The main objective of this project is to enable high-quality wireless communication to vehicular networks in multi-radio access environments. OverDRiVE mainly focuses on Intra Vehicular Area Network (IVAN) environments of private cars and public transportation systems.

OverDRiVE aims to support the movement of IVANs which would connect to different access systems, Mobile Hosts moving in and out of IVANs and the movement of Mobile Hosts within IVANs [43]. The approach taken to handle these network mobility scenarios is by using a MR to enable connectivity for the entire IVAN by extending the MIPv6 protocol. OverDRiVE project relies on the MR-HA bidirectional tunneling approach for basic network mobility support and ensures mobility transparency to the devices within an IVAN.

\section{VIII.C. Nautilus6 (WIDE Project)}

The WIDE (Widely Integrated Distributed Environment) project which was launched in 1988 established the Nautilus6 working group [44] to deploy mobile Internet. In order to do so the Nautilus6 Working Group uses the IETF standards whenever appropriate. Nautilus6 NEMO working group was established in November 2003 and is specifically looking at issues concerned with mobile networks. The main objective of this group is to implement the IETF NEMO Basic protocol.

\section{VIII.D. InternetCAR (Internet Connected Automobile Researches )}

The demand for Intelligent Transportation Systems (ITS) has influenced the need for projects such as the InternetCAR [45]. This project which is also a WIDE project was launched in 1996. Their main aim is to view a car as a node on the Internet and to provide Internet connectivity permanently and in a transparent manner regardless of the underlying access medium.

The InternetCAR project implemented the Prefix Scoped Binding Update approach for network mobility proposed by Ernst et al [46]. The details of this implementation and the requirements for connecting vehicles to the Internet are described in [47].

\section{VIII.E. FleetNet - Internet On the Road}

FleetNet [48] project too aims to connect vehicles to the Internet. But the MR-HA bidirectional tunneling architecture for network mobility is not the approach adapted when providing Internet connectivity to the devices in a vehicle in FleetNet. This project has introduced a novel network mobility architecture named as MOCCA (MObile CommuniCation Architecture).

The MOCCA architecture relies on a new entity namely an Internet Gateway installed on roadsides which plays the role of a gateway router for the devices requiring Internet connectivity. These gateways can be regarded as FleetNet radio nodes since they run the same communication system employed on the vehicles. The Internet Gateways have another interface which connects them to the Internet. The devices in the vehicles are able to obtain connectivity through the Internet Gateways transparently. This MOCCA architecture has been developed by Bechler et al [49].

\section{Conclusion}

This paper presented the basic network mobility architecture using mobility scenarios in order to highlight the benefits of such an architecture. As shown, providing basic network mobility support is relatively simple and requires only minimal extensions to the MIPv6 operations of the MR and its Home Agent. On the other hand, providing route optimization for the nodes within the mobile network is quite challenging, given the MR-HA Bi-directional tunneling architecture. The IETF NEMO working group is chartered only to standardize solutions for basic network mobility; nevertheless numerous members within the community are currently working on optimal routing solutions for a network mobility setting. The future of network mobility at IP level at a large scale relies on the ability to provide advanced mobility support that can coexist with the currently deployed protocols.

\section{ACKNOWLEDGEMENTS}

This work was carried out through an Australian Research Council Linkage Postgraduate Research Award with Vodafone Australia Future Technologies. Special thanks to Robert Hsieh for reviewing this paper on numerous occasions.

\section{REFERENCES}

[1] Perkins C., "IP Mobility support for IPv4," RFC 3220, IETF̈, January 2002.

[2] Johnson D. Perkins C., Arkko J., "Mobility Support in IPv6", (draft-ietf-mobileip-ipv6-24.txt), Internet Draft, IETF, June 30 2003, Work in Progress.

[3] http://www.ietf.org/html.charters/nemocharter.html

[4] Manner J., Kojo M., "Mobiltiy Related Terminology", (draft-ietf-seamoby-mobilityterminology-06.txt), Interent Draft, IETF, february 2004, Work in Progress. 
[5] Ernst T., Lach H-Y., “Network Mobiltiy Support Terminology”, (draft-ietf-nemo-terminology-01), Internet Draft, IETF, February 2004, Work in Progress.

[6] Hager R., Klemets A., Maguire G. Q., Reichert F,; Smith M.T., "MINT- AMobile Internet Router", $1^{\text {st }}$ International Symposium on Global Data Networking,Cairo, Egypt, Dec. 13-15, 1993.

[7] Perkins C.. “IP Mobility Support”, IETF, RFC 2002, October 1996.

[8] Perkins C., "Mobile IP, Design Principles and Practices", Wireless Ćommunications Series, Addison Wesley, 1998. ISBN 0-201-63469-4

[9] Ernst T., "Network Mobiltiy Support in IPv6”, Phd Thesis, University Joseph Fourier Grenoble, France, October 2001.

[10] Ernst T., "Network Mobility Support Goals and Requirements",(draft-ietf-nemo-requirements02.txt), IETF, Internet Draft, Feb. 2004, Work in Progress.

[11] Ernst T., Montavont N., Wakikawa R., Paik E., Ng C., Kuladinithi K., Noel T. "Goals and Benefits of Multihoming”, (draft-multihoming-generic-goalsand-benefits-00), IETF, Internet Draft, Feb. 2004.

[12] Devarapalli V., Wakikawa R., Petrescu A.; $\begin{array}{ll}\text { Thubert P. "NEMO Basic Support } & \text { Protocol" } \\ \text { (draft-ietf-nemo-basic-support-02.txt), } & \text { Internet }\end{array}$ Draft, IETF, December 2003, Work in Progress.

[13] Ferguson P., Senie D., "Network ingress Filtering : Defeating Denial of Service Attacks which Employ IP Source Address Spoofing”, RFC 2267, IETF, January 1998.

[14] Kniveton T. J., Malinen J. T., Devarapalli V., Perkins C. E. "MR Tunneling Protocol" (draftkniveton-mobrtr-03) Internet Draft, IETF, Nov 2002, Work in Progress.

[15] Petrescu A., Catalina-Gallego M., Janneteau C., Lach H.-Y., Olivereau A. "Issues in Designing Mobile IPv6 Network Mobility with the MR-HA Bidirectional Tunnel (MRHA)", (draft-petrescunemo-mrha-02.txt), Internet Draft, IETF, March 2003, Work in Progress.

[16] Thubert P., Molteni M., Ng C., "Taxonomy of Route Optimization models in the Nemo Context", (draft-thubert-nemo-ro-taxonomy-01.txt), Internet Draft, IETF, June 2003, , Work in Progress.

[17] Koodli R., "Fast Handovers for Mobile IPv6" , (draft-ietf-mobileip-fast-mipv6-07.txt), Internet Draft, IETF, September 19 2003, Work in Progress

[18] Hsieh R., Zhou Z. G., Seneviratne A., "S-MIP: A Seamless Handoff Architecture for Mobile IP", In Proceedings of Infocom, San Francisco, US'A, 2003

[19] Droms „R., “DHCPv6 Prefix Delegation for NEMO”, (draft-droms-nemo-dhcpv6-pd-01.txt), Internet Draft, IETF, Feb. 2004, Work in Progress.

[20] Ng C. W., Tanaka T., "Multi-Homing Issues in BiDirectional Tunneling”,(draft-ng-nemomultihoming-issues-00.txt), Internet Draft, IETF, February 2003, Work in Progress.

[21] Wakikawa R., Uehara, K. and T. Ernst, "Multiple Care-of-Address Registration on Mobile IPv6", (draft-wakikawa-mobileip-multiplecoa-01.txt), Internet Draft, IETF, June 2003, Work in Progress.

[22] Wakikawa R., Devarapalli V., Thubert P., "Inter Home Agents Protocol (HAHA)", (draftwakikawa-mip6-nemo-haha-01.txt), Internet Draft, IETF, Feb. 2004, Work in Progress.

[23] Petrescu A., Olivereau A., Janneteau C., Lach HY., "Threats for Basic Netowrk Mobiltiy Support (N'EMO threats)", (draft-petrescu-nemo-threats01.txt), IETF, Internet Draft, January 2004, Work in Progress.
[24] Jung S., Zhao F., Wu F., Kim H., Sohn S., “Threat Analysis for NEMO Basic Operations;, (draftjung-nemo-threat-analysis-02.txt), Internet Draft, IETF, Feb. 2004, Work in Progress.

[25] Faccin S., Le F., Patil B., Perkins C., "Mobile IPv6 Authentication, Authorization and Accounting Requirements", (draft-le-aaa-mipv6-requirements02.txt), Internet Draft, IETF, April 2003, Work in Progress.

[26] Barz C., Frank M., Lach H-Y., Maihoefer C., Petrescu A., Pilz M., Zombik L. "Network Access Control in OverDRIVE Mobile Networks", IST Mobile Summit 2003, Aveiro, Portugal, 16-18 June 2003.

[27] Ernst T., Castelluccia C., Lach H. "Extending Mobile-IPि6 with Multicast to support mobile networks in IPv6”, ECUMN'00, Colmar, France, October 2-4 2000.

[28] Wakikawa R., Koshiba S., Uehara K. "ORC: Optimized Route Cache Management Protocol for Network Mobility" , Proceedings of the $10^{\text {th }}$ Interantional Conference on Telecommunication (ICT), Tahiti Papeete, French Polynesia, Feb 2003.

[29] Jeong J.-H., Lee K.-J., Park J.-S., Kim H.-J. “NDProxy based Route and DNS optimizations for Mobile Nodes in Mobile Network"(draft-jeongnemo-ro-ndproxy-02.txt) Internet Draft, IETF, 14 February 2004, Work in Progress.

[30] Lee K. J., Jeong J. H., Park J.-S., Kim H.-J., "Route Optimization for Mobile Nodes in Mobile Network Based on Prefix Delegation", (draftleekj-nemo-ro-pd-02.txt), Internet Draft, IETF, Feb. 2004, Work in Progress.

[31] Perera E., Hsieh R., Seneviratne A., "Extended Network Mobility Support", (draft-perera-nemoextended-00.txt), Internet Draft, IETF, July 2003, Work in Progress.

[32] Ohinishi H., Sakitani K., Takagi Y., "HMIP based Route Optimization method in a mobile network”, (draft-ohinishi-nemo-ro-hmip-00.txt), IETF, Internet Draft, Oct. 2003, Work in Progress.

[33] Perera E., Seneviratne A., Sivaraman V., "OptiNets: An architecture to enable optimal routing for network mobility ", Accepted for publication, International Workshop on Wireless Ad-hoc Networks 2004, Oulu, Finland, 31 May -3 June, 2004.

[34] Soliman H., Castelluccia C.,El-Malki K., Bellier L., "Hierarchical Mobile IPv6 mobility mangement (HMIPv6)", (draft-ietf-mobileiphmipv6-08.txt), June 2003.

[35] Thubert P., Molteni M., "IPv6 Reverse Routing Header and its application to Mobile Networks, (draft-thubert-nemo-reverse-routing-header-

04.txt), Internet Draft, IETF, Feb. 2004, Work in Progress.

[36] Ng C. W., Tanaka T., "Securing Nested Tunnels Optimization with Access Router Option", (draftng-nemo-access-router-option-00.txt), Internet Draft, IETF, October 2002, Work in Progress.

[37] Na J., Cho S., Kim C., Lee S., Kang H., Koo C., "Secure Nested Tunnels Optimization using Nested Path Information”, (draft-na-nemo-nestedpath-info-00.txt), Internet Draft, IETF, September 2003, Work in Progress.

[38] http://www.ocean.cse.unsw.edu.au/

[39] http://www.nicta.com.au/

[40] http://www.ocean.cse.unsw.edu.au/emotion/index. html

[41] OverDRiVE: http://www.ist-overdrive.org

[42] DRiVE : http://www.ist-drive.org/index2.html

[43] Ronai M., Petrescu A., Tönjes R., Wolf M., "Mobility Issues inOverDRIVE Mobile 
Networks”, IST Mobile Summit 2003, Aveiro, Portugal, 16-18 June 2003.

[44] http://www.nautilus6.org/

[45] InternetCAR:

http://www.sfc.wide.ad.jp/InternetCAR/

[46] Ernst T., Olivereau A., Bellier L., Castelluccia C., Lach H. "Mobile Networks Support in Mobile IPv6” (draft-ernst-mobileip-v6-network-03) Internet Draft, IETF, March 2002, Work in Progress.

[47] Ernst T., Mitsuya K., Uehara K., "Network Mobility from the InternetCAR perspective", Journal Of Interconnection Networks (JOIN), June 2003.

[48] FleetNet : http://www.fleetnet.de/

[49] Bechler M., Franz J., Wolf L., "Mobile Internet Access in FleetNet", 13. Fachtagung Kommunikation in Verteilten Systemen (KiVs 2003), Leipzig, February 2003. 